\title{
A Review in Molar Incisor Hypomineralization
}

\author{
Karine Takahashi* and Heitor Ceolin Araujo \\ Department of Pediatric Dentistry, West Sao Paulo University, Brazil
}

*Corresponding author: Karine Takahashi, Department of Pediatric Dentistry, Profesor at West Sao Paulo University, Francisco Scardazzi Street number 350, Sao Judas Park, Zip code 19023190, Presidente Prudente city, Sao Paulo State, Brazil

Submission: 望 May 23,2018; Published: 望 June 14, 2018

\section{Introduction}

Enamel defects can be observed in both permanent and deciduous teeth. A great variety of terms are used referring to hypomineralized teeth, although in 2001, Weerheijm et al. named this alteration as "Molar Incisor Hypomineralization", which is described as enamel degeneration involving first permanent molars and also or not, permanent incisors. Dental enamel has ectodermic origin and is highly mineralized and very sensible to systemic alterations. Enamel defects can cause permanent flaws in erupted teeth, caused by lack of metabolic activity. Besides, teeth diagnosed with MIH differ in chemical composition and less enamel. Probably these kind of defect. Has multifactorial etiology, such as local factors, genetic can affect enamel development. Some authors associated etiology to systemic origin, but it is not completely understood. Somewhat disturbance during amelogenesis cause by predisposition of genetics and environmental effects from the first 3 months of pregnancy until 3 years of old can cause MIH. Potential causative factors in this age are respiratory diseases, breastfeeding, prolonged use of medicaments and maternal smoking. Several and repeated medical conditions also are associated with development of MIH. Laboratory studies in rats suggest that exposure to bysphenol A can cause enamel defects, which targets two genes responsible for enamel matrix degradation.

Affection of two specific types of teeth, molars and incisors, suggest a specific window of time of disturbance occurring during maturation stage of affected teeth. MIH has a high prevalence worldwide, however countries with medium to low incomes are the most affected, it ranges between $2,4 \%$ to $40,2 \%$. Some authors related this variation to medical conditions related to birth or after birth. Prevalence variations depend on country, region or age group. Needs of treatment in MIH are described as increase of sensitivity, caries lesions progress, aesthetic, and also poor development of mastigatory efforts. Treatment is complex, requiring planning and regular maintenance, as restorative treatment has an unfavorable prognosis, difficulties regarding management of pain and behavior. Teeth affected have increase in caries experience, need to restoration replacement and in seriously cases, extraction and orthodontic treatment. For all the reasons listed above, treatment and diagnosis are confusing for clinicians related to etiology, aspect of lesions and as a recent disease, which become a challenge for Dentistry in the recent years.
Creative Commons Attribution 4.0 International License

For possible submissions Click Here

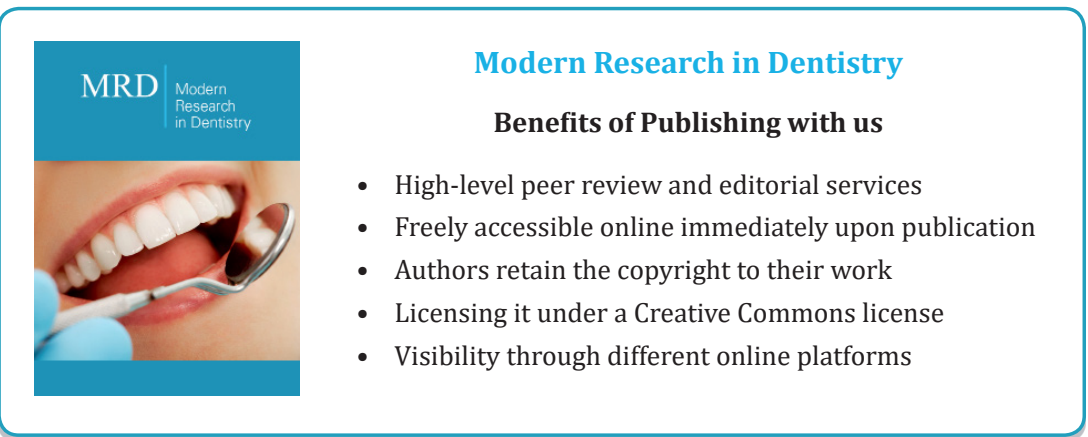

